\title{
Propiedades psicométricas de las escalas de variables disposicionales y contextuales (IEPA y AYES) adaptadas a la asignatura Inglés como Lengua Extranjera
}

\author{
Beatriz Sala-Álvarez del Manzano', Ana Isabel Álvarez-González² y \\ Carmen González-González de Mesa ${ }^{2 *}$ \\ 1 Consejería de Educación y Cultura del Principado de Asturias. \\ 2 Universidad de Oviedo
}

\begin{abstract}
Resumen: Los objetivos de este estudio han sido: a) examinar la validez de la escala de Variables Disposicionales (IEPA), que evalúa el interés, progresión y el esfuerzo del alumnado, y la escala de Variables Contextuales (AYES) que evalúa si el docente ayuda a aprender, valora el esfuerzo y despierta interés, para la asignatura Inglés como Lengua Extranjera en Educación Secundaria, Bachillerato y Formación Profesional de Grado Medio y Superior; b) estudiar si las variables personales tienen un efecto sobre los factores de las escalas; y c) determinar si los factores contextuales pueden predecir los factores disposicionales. Participaron 1086 estudiantes de colegios públicos y concertados de Oviedo (España). Se les aplicó un cuestionario compuesto por las escalas y por preguntas de tipo personal y familiar. El análisis estadístico se realizó con los programas FACTOR. 10 y SPSS.24. Los resultados del análisis de la estructura factorial y la consistencia interna fueron adecuados para ser utilizados en el contexto de la asignatura Inglés como Lengua Extranjera. La percepción del alumnado sobre el contexto de enseñanza, en términos de apoyo proporcionado, valoraciones positivas y motivación, predicen el interés de los estudiantes, la progresión y el esfuerzo hacia la asignatura.
\end{abstract}

Palabras clave: Validación; IEPA; AYES; Contexto de aprendizaje; Disposición al estudio.

\section{Psychometric properties of the Scales of Dispositional and Contextual Variables (IEPA and AYES) adapted to English as a Foreign Language subject}

\begin{abstract}
This study aims to test the validity of the Dispositional Variables (IEPA) scale, which evaluates students' learning interest, progress, and effort, and of the Contextual Variables (AYES) scale, which evaluates whether the teacher helps students to learn, values theireffort, and can arouse their interest in the subject English as a Foreign Language in Compulsory Secondary Education and Higher Secondary Education. Other aims are to determine whether personal variables affect the scale factors and whether contextual variables can predict dispositional variables. Participants were 1086 students from public and private schools in Oviedo (Spain) who completed a questionnaire composed of the scales, and which also collected personal and family data. The statistical analysis was carried out using the programs FACTOR. 10 and SPSS.24. The results of the analysis of the factor structure and internal consistency showed that the scales were suitable to be used in the context of the English as a Foreign Language subject. Students' perception of the teaching context, in terms of support provided, positive assessments, and motivation, predict their interest, progress, and effort made in the subject of English.
\end{abstract}

Keywords: Validation; IEPA; AYES; Learning context; Disposition to study.

En España el preámbulo de la Ley Orgánica de la Calidad Educativa (2002) incide en el objetivo de que los estudiantes lleguen a

Recibido: 29/07/2020 - Aceptado: 22/10/2020 - Avance online: 01/12/2020 *Correspondencia: Carmen González González de Mesa.

Facultad de Formación del Profesorado y Educación. Aniceto Sela S/N Dirección: 33005, Oviedo, España.

E-mail: gmcarmen@uniovi.es

Sala-Álvarez del Manzano, B., Álvarez-González, A. I. y González-González de Mesa, C. 2021). Propiedades psicométricas de las escalas de variables disposicionales y contextuales (IEPA y AYES) adaptadas a la asignatura Inglés como Lengua Extranjera. Revista de Psicología Educación, 16(1), 18-30, https://doi.org/10.23923/rpye2021.01.199 desenvolverse con fluidez en al menos una primera lengua extranjera, y considera que el dominio de una segunda lengua extranjera se muestra como una de las principales carencias de nuestro sistema educativo, apostando por la incorporación curricular de una segunda lengua extranjera. Mejorar los distintos aspectos implicados en la práctica docente y

1699-9517/@ 2021 Asociación Científica de Psicología y Educación (ACIPE). Publicado por Consejo General de Colegios Oficiales de Psicólogos, España. Este es un artículo Open Access bajo la CC BY-NC-ND licencia (http://creativecommons.org/licencias/bync-nd/4.0/. 
en el proceso de aprendizaje, en particular las variables afectivas, contribuirá positivamente a la mejora de la competencia comunicativa de los estudiantes en lengua extranjera. En este estudio se pretende medir la motivación y el interés del alumnado adolescente hacia la asignatura de Inglés como Lengua Extranjera, así como la reflexión sobre su aprendizaje, con el fin de buscar mejorar aquellos aspectos que pueden ser perfeccionados.

Diversas teorías destacan la influencia que ejercen sentimientos y emociones personales en el aprendizaje de nuevos contenidos, como la teoría de "aprendizaje y orientación", o Counseling-Learning (Curran, 1976), que considera fundamental el apoyo al alumnado por encima del contenido lingüístico y afirma que un ambiente relajado en el aula es fundamental, siendo el docente guía en todo el proceso de aprendizaje. Para este autor, el profesorado es meramente el consejero - guía del alumnado para que este supere las barreras psicológicas que dificultan su aprendizaje. El aprendizaje se debe a un proceso de maduración y, por este motivo, esta teoría tiene en cuenta los factores emocionales que inciden en el aprendizaje del alumnado, siendo tan importantes los factores cognitivos como los afectivos, concediendo gran relevancia a los sentimientos.

Otras teorías, como la llamada de "filtro afectivo" (Krashen, 1982), indican que los factores afectivos, tales como la ansiedad y la motivación, actúan en el proceso de adquisición de una segunda lengua y defienden que, si la persona está motivada y se siente segura de sí misma, el proceso de adquisición de nuevos conocimientos se verá reforzado. Un alto grado de ansiedad, por ende, puede ralentizar el proceso de la adquisición del idioma a aprender.

La motivación es uno de los factores que determinan el comportamiento humano y nos dirige a una acción. El Diccionario de la Real Academia Española (2001) define la motivación como el conjunto de factores internos y externos que determinan las acciones de una persona. La palabra motivación proviene del verbo latino movere: nos movemos o actuamos para lograr aquello que queremos alcanzar. La motivación es, pues, el porqué de la conducta, las necesidades o deseos que dirigen el comportamiento y explican lo que hacemos (Nevid, 2013).

Es difícil determinar si la actitud positiva produce un aprendizaje exitoso, o si el aprendizaje exitoso origina actitudes positivas, o si ambos son influenciados por otros factores. Sin embargo, hay una amplia evidencia de que la motivación positiva está relacionada con la voluntad de seguir aprendiendo (Barragan, Colcha, \& Herrera, 2019; Lightbown \& Spada, 2013). Para Gardner, Tremblay y Masgoret (1997), la motivación que producen las actitudes hacia la asignatura de lengua crea autoconfianza, además de generar el aprendizaje de estrategias, y tanto la motivación como la aptitud y uso de las estrategias motivan el aprendizaje. La motivación, a veces, se asocia con factores afectivos de la personalidad y de la emoción (Crookes \& Scmidt, 1991) y hay quienes creen que los docentes han de incluirla en sus currículos, porque así se lograrían mejores resultados y el aprendizaje en el aula sería más efectivo (Dörney, 2001). Otros autores señalan la importancia de la actividad lúdica como medio motivador del aprendizaje y la atención (Muñoz, Lira, Lizama, Valenzuela, \& Sarlé, 2019), distinguiendo la importancia de las experiencias satisfactorias que los futuros docentes experimentan durante su formación, para potenciar su capacidad creativa, la cual les ayudará al desarrollo de los recursos educativos. Es necesario que el profesorado en formación desarrolle la motivación intrínseca para sentirse más autónomos a la hora de proyectar al labor docente (Burgueño, Sicilia, Alcaraz-lbáñez, \& Lirola, 2020).

No cabe duda de que incidir positivamente en la motivación del alumnado es uno de los retos más atrayentes, y a la vez más complicados, a los que se enfrentan los docentes hoy en día, siendo esencial el papel del profesorado en la motivación del alumnado, no solo a través de los comentarios y sugerencias que los docentes hacen acerca de las actividades de sus estudiantes, sino también al establecer metas de carácter cooperativo que incrementan la 
autoconfianza y les hace percibir que tienen una tarea importante que desarrollar y que sus compañeros cuentan con ellos (Crookes \& Scmidt, 1991).

La mayoría de los trabajos de investigación que estudian el efecto que las variables afectivas ejercen en el aprendizaje de segundas lenguas en el alumnado de secundaria están fundamentalmente relacionados con el aprendizaje del inglés (Riemer \& Schlak, 2004).

La investigación acerca de la motivación y su relación con el aprendizaje de una lengua extranjera se remonta a los años 50 del siglo XX (Rost-Roth, 2001), siendo Gardner (1985), quien investigó el papel de las variables afectivas, como pueda ser la motivación, en el aprendizaje de una segunda lengua. El interés por el estudio de las variables afectivas en relación con el aprendizaje de segundas lenguas tuvo su mayor auge en la década de los años 90, periodo en el que se llevaron a cabo investigaciones para analizar la correlación entre la motivación y el estudio del inglés, destacando Madrid (1999) y Tragant \& Muñoz (2000), que llegan a la conclusión de que las variables afectivas, como la motivación, favorecen no solo el aprendizaje de segundas lenguas, sino también el empeño de querer comunicarse en la lengua objeto de estudio. En los últimos años, otros autores (Bastidas \& Muñoz, 2020) también han considerado que el ambiente de aprendizaje creado en el aula tiene una influencia importante en el aprendizaje del inglés y Gabarrón (2019) afirma que el profesorado de lenguas extranjeras debe ser emocionalmente inteligente e interactuar con su alumnado para trasmitir la enseñanza de la lengua desde la afectividad y la positividad, y de este modo desarrollar la inteligencia emocional.

Sánchez (2017) ha demostrado que algunos programas, entre los que se encuentra la programación neurolingüística, fortalecen no solo las habilidades comunicativas del docente, sino también la capacidad para educar al alumnado a nivel emocional para que su aprendizaje sea de mayor calidad. GómezOrtiz, Romera, \& Ortega-Ruiz (2017) sugieren que en los centros escolares se preste atención a las emociones, ya que así se beneficia y se ayuda al alumnado a encontrar un espacio donde se sientan amparados y apoyados por el resto de los compañeros.

Guillen, Pérez-Luzardo, \& Arnaiz (2013) llegan a la conclusión de que para que el aprendizaje de una lengua extranjera sea exitoso es importante no solo mantener el nivel de motivación del alumnado, algo ya manifestado por autores como Mas and Medinas (2007), sino también el nivel de optimismo.

A la vista de las investigaciones realizadas hasta el momento, el hecho de adaptar, con garantía de éxito, unas escalas que evalúen la disposición de los estudiantes en la asignatura Inglés como Lengua Extranjera y los contextos donde se desarrolla el proceso de aprendizaje, puede ser relevante para potenciar en el alumnado adolescente la motivación necesaria para aumentar el interés, potenciar el esfuerzo y progresar en el aprendizaje de una lengua extranjera.

Así las cosas, los objetivos de este trabajo han sido, en primer lugar, validar las escalas de Variables Disposicionales (IEPA) y Variables Contextuales (AYES) en el ámbito de la asignatura Inglés como Lengua Extranjera; en segundo lugar, estudiar si las variables personales, edad y sexo, pueden tener un efecto sobre los factores a estudiar; y, en tercer lugar, determinar si las variables contextuales pueden ser predictoras de las variables disposicionales. La escala IEPA evalúa el interés, esfuerzo y progresión en el aprendizaje del alumnado preadolescente y adolescente (12 a 20 años) y la escala AYES si el docente les ayuda a aprender, valora el esfuerzo y despierta interés por la asignatura. Cabe esperar que se cumpla esta estructura factorial.

\section{MÉTODO}

\section{PARTICIPANTES}

Los participantes en el estudio han sido estudiantes de Educación Secundaria Obligatoria (ESO), Bachillerato y Formación Profesional de Grado Medio y Superior, matriculados en centros, tanto públicos como concertados, de Oviedo (España), seleccionados por conveniencia. Los 
cuestionarios fueron aplicados a 1097 personas, de los que se han suprimido 11 por no haber sido cumplimentados correctamente dejando ítems sin contestar. Participaron en el estudio seis centros escolares, tres de ellos públicos y tres concertados, todos situados en zona urbana, pero con diferentes características, ya que, aunque todos ellos cuentan con Educación Secundaria Obligatoria, tan solo tres imparten Formación Profesional y únicamente uno de los centros oferta el Bachillerato Internacional. En cuanto al alumnado participante, 586 son varones y 500 mujeres, con edades comprendidas entre los 12 y los 20 años $(M=14.82$, DT = 1.88). El ámbito de estudio abarca los cuatro cursos de ESO, los dos cursos de Bachillerato, los dos de Formación Profesional de Grado Medio y los dos de Formación Profesional de Grado superior.

\section{INSTRUMENTOS DE MEDIDA}

Las escalas a validar para su aplicación en la asignatura de Inglés, han sido la escala de evaluación de las Variables Disposicionales (IEPA), que valora la disposición de los estudiantes hacia la asignatura (interés, esfuerzo y progresión en el aprendizaje) y consta de 12 ítems; y la escala de evaluación de las Variables Contextuales (AYES), que valora en el contexto de la clase si el docente ayuda en el aprendizaje, despierta interés y valora el esfuerzo de los estudiantes, también con 12 ítems. Las opciones de respuesta siguen una escala de respuesta tipo Likert de 5 puntos (1 totalmente en desacuerdo, 5 totalmente de acuerdo con las afirmaciones presentadas). En ambas escalas los ítems van precedidos por la frase "En la clase de la asignatura de Inglés como Lengua Extranjera...". Los ítems de las escalas pueden verse en las tablas 1 y 3 .

Estas dos escalas han sido validadas por Cecchini et al. (2014) en el contexto de las asignaturas de Matemáticas, Lengua y Literatura y Educación Física, partiendo de las escalas de orientación motivacional de Duda \& Nicholls (1992) y adaptadas y traducidas a la versión española por Balaguer, Castillo, \& Duda (2001).
Dado que cada escala consta de 12 ítems, podría cubrirse en 15 minutos, por lo que se considera eficaz para ser aplicadas a escolares en edades preadolescentes y adolescentes, sin que lleguen a sucumbir en el aburrimiento.

\section{PROCEDIMIENTO}

En primer lugar, se solicitó permiso a la Facultad de Formación del Profesorado y Educación de la Universidad de Oviedo, en base al convenio con la Consejería de Educación y Cultura del Principado de Asturias, para llevar a cabo el estudio en centros escolares. Una vez obtenido, se contactó con la dirección de los centros educativos para explicarles el objetivo de la investigación, su estructura y contenido, y solicitarles autorización y colaboración para proceder al trabajo de campo. Conseguido su visto bueno, y el consentimiento informado de las familias de los estudiantes, se estableció un calendario de visitas a los centros. El cuestionario fue aplicado por una persona entrenada para tal efecto, en un aula y en sesión única para cada grupo, y siempre dentro del horario lectivo.

Tras explicaralalumnadoelobjeto del estudio y pedir su colaboración, garantizándoles el anonimato y la confidencialidad de los datos para la investigación y posterior difusión de los resultados, el tiempo empleado estuvo en torno a los 15 minutos. Se insistió en la importancia de contestar a todos los ítems, con el fin de que el estudio pudiese realizarse de manera adecuada. La duración del periodo de toma de datos ha sido de dos meses.

\section{ANÁLISIS DE DATOS}

El análisis estadístico realizado para la investigación instrumental comnenzó con el cálculo de los estadísticos univariados para cada ítem de las escalas: media, desviación típica, asimetría, curtosis e índice de homogeneidad corregida. Seguidamente se procedió a analizar la dimensionalidad de las escalas mediante un análisis factorial exploratorio (AFE) o semiconfirmatorio. 
Para la realización del AFE se utilizó el método de Implementación Óptima de Análisis Paralelos, propuesto por Timmerman y LorenzoSeva (2011), para especificar el número de factores a partir de correlaciones policóricas entre variables, llevándose a cabo 10000 remuestreos. Para la factorización se aplicó el método de mínimos cuadrados no ponderados, utilizando para ello el método Promin de rotación (Finn \& Rock, 1997; Ferrando \& Lorenzo-Seva, 2014). Para el cálculo de fiabilidad se aplicó el coeficiente alfa de Cronbach para datos ordinales (Elosua \& Zumbo, 2008).

En la investigación causal-comparativa se utilizaron estadísticos paramétricos, condicionados por los valores que se obtengan de los índices de asimetría y curtosis, atendiendo las advertencias de Schmider, Ziegler, Danay, Beyer, \& Bühner (2010), que explican que los estadísticos de Kolmogolov-Smirnov con la corrección de Lilliefors y de Shapiro Wilk son extremadamente sensibles, por tanto, si en una muestra amplia los valores de asimetría y curtosis no superan los \pm 2.0 y \pm 4.00 respectivamente de la desviación de la normalidad, los estadísticos paramétricos son suficientemente robustos.

Se han realizado pruebas de tendencia central y dispersión para todos los factores resultantes y análisis bivariado entre los factores mediante el coeficiente de correlación de Pearson. A continuación, se realizaron los MANOVA(s), tomando el sexo y la edad como variables independientes, y los factores disposicionales y contextuales como variables dependientes. También se realizó un análisis de regresión simple, para comprobar si las variables contextuales son predictoras de las variables disposicionales y con qué peso.

Los programas estadísticos utilizados han sido el FACTOR 10.3 para realizar el análisis factorial y el SPSS. 24 para el cálculo de fiabilidad y el estudio causal-comparativo.

\section{RESULTADOS}

Se presentarán seguidamente los resultados del análisis instrumental de las escalas IEPA y AYES.

Con respecto a la escala IEPA, se exponen los resultados del cálculo de los valores descriptivos de cada uno de los ítems (Tabla 1). Todos muestran una desviación típica superior a 1; una asimetría y una curtosis entre \pm 1.00 y un índice de homogeneidad corregida superior a .50, por tanto la distribución muestral es aceptable (Bandalos \& Finney, 2010; Ferrando \& Anguiano-Carrasco, 2010; Forero, MaydeuOlivares,\& Gallardo-Pujol, 2009; Kline, 1999; Muthén \& Kaplan, 1992).

Tabla 1

Medidas de tendencia central y dispersión de las variables de la escala IEPA

\begin{tabular}{|c|c|c|c|c|c|}
\hline En las clases de inglés: & M & DT & Asi. & Cur. & $\mathrm{IHC}$ \\
\hline 1. El aprendizaje es divertido & 3.05 & 1.469 & -0.178 & -0.820 & .639 \\
\hline 2. Noto que mejora mi nivel & 3.49 & 1.467 & -0.583 & -0.576 & .743 \\
\hline 3. Persisto en el intento de mejorar & 3.87 & 1.000 & -0.888 & 0.626 & .619 \\
\hline 4. Lo paso bien aprendiendo & 3.25 & 1.383 & -0.317 & -0.643 & .688 \\
\hline 5. Observo que mejoran mis habilidades en esta asignatura & 3.53 & 1.307 & -0.619 & -0.346 & .774 \\
\hline 6. Me esfuerzo por aprender & 3.87 & 1.003 & -0.783 & 0.238 & .624 \\
\hline 7. Me divierto aprendiendo & 3.22 & 1.378 & -0.301 & -0.673 & .666 \\
\hline 8. Veo que estoy mejorando & 3.59 & 1.276 & -0.636 & -0.313 & .769 \\
\hline 9. Me exijo todo lo que puedo & 3.44 & 1.348 & -0.427 & -0.541 & .537 \\
\hline 10. El aprendizaje es interesante y entretenido & 3.10 & 1.407 & -0.198 & -0.775 & .694 \\
\hline 11. Siento que avanzo & 3.55 & 1.338 & -0.642 & -0.344 & .761 \\
\hline 12. Intento superarme & 3.86 & 1.123 & -0.838 & 0.234 & .628 \\
\hline
\end{tabular}


Calculado el estadístico de Bartlett [9577.3 $(D f=66 ; p \leq .000010)]$ y el test de KaiserMeyer-Olkin (KMO =.91), se puede observar un buen ajuste de los datos, lo cual permite proceder al análisis factorial. El carácter ordinal del registro de los datos -escala Likert de 1 a 5 revela que la extracción de factores en el AFE conviene realizarla aplicando la estimación robusta de mínimos cuadrados no ponderados (ULS) a partir de la matriz de correlaciones policóricas.

Se procede a realiza el análisis factorial. Los tres factores extraídos explican el $82 \%$ de la varianza. El índice de ajuste comparado (CFI) y el índice de bondad de ajuste (GFI), superan el .95, siendo ambos valores de 99. Se ha calculado la raíz cuadrática media de los residuales (RMSR) obteniendo un valor de .05. Los datos extraídos, muestran por lo tanto un ajuste razonable de la estructura tridimensional para estos ítems.
A continuación, se presentarán los resultados de la rotación (Promin), que explican las matrices de las cargas rotadas en tres factores y, además, el resultado de la media y desviación típica de cada factor, el coeficiente de fiabilidad (Alfa de Cronbach) y la correlación interfactorial (Tabla 2). El Factor 1 se corresponde con Interés hacia la Asignatura, el Factor 2 con Progresión en el Aprendizaje y el Factor 3 con Esfuerzo por Aprender. El factor que presenta una media más alta es Esfuerzo por Aprender ( $M=3.76)$, seguido de Progresión en el Aprendizaje $(M=$ 3.54) y el más bajo puntuado es el de Interés hacia la Asignatura $(M=3.15)$. El índice de fiabilidad es favorable, superior a .70 en los tres factores: Interés hacia la Asignatura ( $\alpha$ $=.940)$, Progresión en el Aprendizaje ( $\alpha=$ .890) y Esfuerzo por Aprender ( $\alpha=$.850). La correlación interfactorial presenta puntuaciones superiores a .50 .

Tabla 2

Matriz Factorial Rotada (IEPA) Medias, Alfa de Cronbach y Correlación interfactorial

\begin{tabular}{|c|c|c|c|c|}
\hline & & $\mathrm{F} 1$ & $\mathrm{~F} 2$ & F3 \\
\hline V 1 & & .224 & .746 & -.182 \\
\hline V 2 & & .974 & .026 & -.089 \\
\hline V 3 & & .204 & .029 & .583 \\
\hline V 4 & & -.006 & .893 & -.006 \\
\hline V 5 & & .868 & .058 & .021 \\
\hline V 6 & & -.041 & .117 & .779 \\
\hline V 7 & & -.116 & .938 & .049 \\
\hline V 8 & & .906 & .006 & .035 \\
\hline V 9 & & -.053 & -.069 & .873 \\
\hline V 10 & & .139 & .777 & -.048 \\
\hline V 11 & & .953 & -.065 & .051 \\
\hline V 12 & & -.029 & -.049 & .951 \\
\hline $\begin{array}{c}\text { Media y Desviación típica } \\
\text { de cada factor }\end{array}$ & & 3.15 (1.029) & $3.54(1.069)$ & $3.76(.878)$ \\
\hline $\begin{array}{l}\text { Coeficiente de fiabilidad } \\
\text { ( } \alpha \text { de Cronbach) }\end{array}$ & & .940 & .890 & .850 \\
\hline \multirow{3}{*}{$\begin{array}{l}\text { Correlación } \\
\text { interfactorial }\end{array}$} & F1 & - & .649 & .645 \\
\hline & F2 & - & - & .599 \\
\hline & F3 & - & - & - \\
\hline
\end{tabular}


Una vez comprobada la validez de constructo (la estructura factorial) de la escala IEPA y que se corresponde con el trabajo de validación de los creadores de la escala (Cecchini et al., 2014), se procedió a comprobar si es también apropiada la escala AYES.

Los valores del análisis descriptivo de cada uno de los ítem muestran también una distribución conveniente: desviación típica superior a 1, asimetría y curtosis entre \pm 1 , e índice de homogeneidad corregido superior a.50 (Bandalos \& Finney, 2010; Ferrando \& Anguiano-Carrasco, 2010; Forero, MaydeuOlivares, \& Gallardo-Pujol, 2009; Kline, 1999; Muthén \& Kaplan, 1992). Pueden verse los resultados en la Tabla 3.
Se ha calculado el estadístico de Bartlett [8134.1 (Df =66; $p \leq .000010)]$ y el test de Kaiser-Meyer-Olkin (KMO = .93), observando un buen ajuste de los datos, lo cual admite realizar el análisis factorial.

Los tres factores extraídos explican el 77\% de la varianza. Los valores de CFI y GFI ambos han sido $=.99$; y el valor de RMSR $=.05$, datos que muestran un buen ajuste para una estructura de tres factores.

Los resultados de la rotación (Promin), muestran tres factores, el Factor 1 se corresponde con que el profesorado de inglés Despierta Interés, el Factor 2 con que Ayuda en el Aprendizaje y el Factor 3 con que Valora el Esfuerzo. La media más alta la alcanza el

Tabla 3

Medidas de tendencia central dispersión de las variables de la escala AYES

\begin{tabular}{|c|c|c|c|c|c|}
\hline En las clases de inglés: & M & DT & Asi. & Cur. & $\mathrm{IHC}$ \\
\hline 1. Las propuestas del profesorado son divertidas & 2.95 & 1.183 & -0.105 & -0.826 & .642 \\
\hline 2. El profesorado nos ayuda cuando tenemos problemas para aprender & 3.86 & 1.096 & -0.926 & 0.264 & .654 \\
\hline 3. Se recompensa el esfuerzo y la participación de los estudiantes & 3.53 & 1.148 & -0.564 & -0.377 & .672 \\
\hline 4. Las actividades y tareas que se hacen en clase son agradables & 3.25 & 1.128 & -0.366 & -0.520 & .711 \\
\hline 5. El profesorado nos guía adecuadamente en el aprendizaje & 3.64 & 1.089 & -0.698 & -0.048 & .748 \\
\hline 6. Se tiene en cuenta el esfuerzo y la constancia del alumnado & 3.62 & 1.175 & -0.686 & -0.318 & .734 \\
\hline 7. Las tareas y lecciones despiertan el interés del alumnado & 2.87 & 1.100 & -0.054 & -0.635 & .635 \\
\hline 8. El profesorado sabe cómo ayudarnos y lo hace & 3.59 & 1.081 & -0.597 & -0.175 & .762 \\
\hline 9. Se valora la participación y el esfuerzo del alumnado & 3.78 & 1.096 & -0.778 & -0.020 & .689 \\
\hline 10. La oferta de actividades es interesante y sugestiva & 3.06 & 1.126 & -0.141 & -0.619 & .646 \\
\hline 11. El profesorado nos enseña a aprender & 3.61 & 1.158 & -0.645 & -0.353 & .698 \\
\hline 12. Se reconoce el esfuerzo del alumnado & 3.66 & 1.186 & -0.688 & -0.337 & .729 \\
\hline
\end{tabular}


factor de Ayuda en el Aprendizaje y la más baja en que Despierta Interés. El análisis de fiabilidad alcanza valores superiores a .70 en los tres factores: el profesorado de Inglés Demuestra Interés ( $\alpha=.858)$, Ayuda en el Aprendizaje ( $\alpha=.866$ ) y Valora el Esfuerzo ( $\alpha$ $=.887)$. La correlación interfactorial presenta puntuaciones superiores a .70. Ver Tabla 4.

Una vez comprobada la validez de la estructura factorial de las dos escalas para que pueda aplicarse en la asignatura con garantía de éxito, se procedó al estudio causalcomparativo. Primeramente, se clasificó a los estudiantes en dos grupos: preadolescentes hasta los 14 años, que se corresponde con el primer ciclo de $\mathrm{ESO}$; y adolescentes con más de 14 años, que abarca segundo ciclo de la ESO, Bachillerato y Formación profesional.

Se comienza estudiando los resultados inferenciales de la escala IEPA realizando un análisis multivariado de varianza (MANOVA), considerando como variables independientes el sexo y edad dividida en preadolescentes y adolescentes $(2 \times 2)$, y como variables dependientes los factores resultantes (Interés hacia la Asignatura, Progresión en el Aprendizaje y Esfuerzo por Aprender).

EI MANOVA reveló un efecto principal significativo para el sexo $\left(\Lambda=.966, \mathrm{~F}_{(3,1080)}=\right.$ $\left.12.644, p<.001, \eta^{2}=.034,1-\beta=1.000\right)$, para la edad $\left(K=.938, F_{(3,1080)}=23.674\right.$, $\left.p<.001, \eta^{2}=.062,1-\beta=1.000\right)$ y para

Tabla 4

Matriz Factorial Rotada (AYES), Medias, alfa de Cronbach y correlación interfactorial

\begin{tabular}{|c|c|c|c|c|}
\hline & & F1: DEM.INT & F2: AYU.APR & F3: VAL.ESF \\
\hline V1 & & .890 & .078 & -.181 \\
\hline V2 & & -.144 & .957 & -.061 \\
\hline V3 & & .019 & .099 & .685 \\
\hline V4 & & .778 & .038 & .037 \\
\hline V5 & & .080 & .796 & -.019 \\
\hline V6 & & .013 & .008 & .862 \\
\hline V7 & & .827 & -.091 & .040 \\
\hline V8 & & -.096 & .897 & .068 \\
\hline V9 & & -.016 & -.039 & .904 \\
\hline V10 & & .802 & .054 & -.078 \\
\hline V11 & & .025 & .755 & .025 \\
\hline V12 & & -.002 & -.036 & .927 \\
\hline $\begin{array}{l}\text { Media y Desviación típica de } \\
\text { cada factor }\end{array}$ & & $\begin{array}{c}3.02 \\
(.950) \\
\end{array}$ & $\begin{array}{l}3.67 \\
(.934) \\
\end{array}$ & $\begin{array}{l}3.65 \\
(.995) \\
\end{array}$ \\
\hline $\begin{array}{l}\text { Coeficiente de fiabilidad } \\
\text { ( } \alpha \text { de Cronbach) }\end{array}$ & & .858 & .866 & .887 \\
\hline \multirow{3}{*}{$\begin{array}{l}\text { Correlación } \\
\text { interfactorial }\end{array}$} & $\mathrm{F} 1$ & - & .827 & .711 \\
\hline & F2 & - & - & .823 \\
\hline & F3 & - & - & - \\
\hline
\end{tabular}


la interacción sexo $x$ edad $\kappa=.987, \mathrm{~F}_{(3,1080)}$ $=4.878, p<.01, \eta^{2}=.013,1-\beta=.909$. Los posteriores análisis univariados (ANOVA) han revelado que las mujeres muestran valores significativamente más altos en Interés hacia la Asignatura $\left(\mathrm{F}_{(1,1085)}=6.945, \mathrm{p}<.01, \eta^{2}=.009\right.$, $1-\beta=.750)$ y Esfuerzo por Aprender $\left(F_{(1,1085)}\right.$ $\left.=24.935, p<.001, \eta^{2}=.023,1-\beta=.999\right)$. En cuanto a la edad, los análisis muestran que puntúan estadísticamente significativo más alto los preadolescentes que los adolescentes en los tres factores: Interés hacia la Asignatura $\left(F_{(1,1085)}\right.$ $\left.=11.674, p<.01, \eta^{2}=.011,1-\beta=.927\right)$, Progresión en el Aprendizaje $\left(F_{(1,1085)}=59.847\right.$, $\left.p<.001, \eta^{2}=.052,1-\beta=1.000\right)$ y Esfuerzo por Aprender $\left(F_{(1,1085)}=40.664, p<.001, \eta^{2}=\right.$ $.035,1-\beta=1.000)$.

A continuación, se procederá a presentar los resultados de la escala AYES. Siguiendo el mismo esquema que en la escala anterior, se comienza con los resultados del MANOVA $2 \times 2$, considerando las variables independientes sexo y edad, y como variables dependientes los factores resultantes (el profesorado de inglés Despierta Interés, Ayuda en el Aprendizaje y Valora el Esfuerzo ). Los resultados muestran un efecto estadísticamente significativo con respecto a la edad $\left(K=.973, \mathrm{~F}_{(3,1080)}=9.986\right.$, $\left.p<.001, \eta^{2}=.027,1-\beta=.998\right)$, pero no respecto al sexo y la interacción entre ambas variables. Los análisis univariados revelan que los estudiantes preadolescentes puntúan de forma estadísticamente más alta que los adolescentes: el profesorado de inglés Despierta Interés $\left(F_{(1,1085)}=20.905, p<\right.$ $\left..01, \eta^{2}=.019,1-\beta=.995\right)$ y Ayuda en el Aprendizaje $\left(F_{(1,1085)}=19.038, p<.001, \eta^{2}\right.$ $=.017,1-\beta=.992)$, pero no en el factor referido a que el profesorado de Inglés Valora el Esfuerzo. En cuanto al sexo, aunque las mujeres puntúan más alto en los tres factores, los resultados no presentan diferencias estadísticamente significativas.

Las correlaciones bivariadas (Correlación de Pearson) entre los seis factores resultantes indican una potente correlación positiva entre ellos, siendo todas estadísticamente significativas en un 99\%, y superando en todos los casos el .300.

Se realizó un análisis de regresión para determinar si la percepción que el alumnado tiene sobre el contexto donde se desarrolla la enseñanza del inglés puede predecir de alguna manera la disposición hacia el aprendizaje. Se han utilizado los tres factores de la escala AYES como variables predictivas de los tres factores de la escala IEPA.

Tabla 5

Correlaciones bivariadas entre factores

\begin{tabular}{|c|c|c|c|c|c|}
\hline Factores & 2 & 3 & 4 & 5 & 6 \\
\hline 1.Interés por el aprendizaje & $.613^{* *}$ & $.504^{* *}$ & $.752^{\star \star}$ & $.556 * \star$ & $.461 * *$ \\
\hline 2.Progresión en el aprendizaje & & $.573^{* *}$ & $.584^{\star *}$ & $.525^{\star *}$ & $.438 * \star$ \\
\hline 3.Esfuerzo en el aprendizaje & & & $.426 * *$ & $.418^{* *}$ & $.344^{\star *}$ \\
\hline 4.El profesor demuestra interés & & & & $.683^{* *}$ & $.595^{\star \star}$ \\
\hline 5.El profesor ayuda a aprender & & & & & $.722^{\star \star}$ \\
\hline 6.El profesor valora el esfuerzo & & & & & \\
\hline \multicolumn{6}{|c|}{ **La correlación es significativa en el nivel 0.01} \\
\hline
\end{tabular}


Tabla 6

Porcentajes de varianza única en los factores disposicionales explicada por los factores contextuales

\begin{tabular}{|c|c|c|c|c|c|}
\hline $\begin{array}{c}\text { Variables } \\
\text { dependientes }\end{array}$ & $\begin{array}{l}\text { Variables } \\
\text { predictoras }\end{array}$ & B & $\mathrm{R}^{2}$ & $F$ & $p$ \\
\hline \multirow{3}{*}{ INTER } & DES.INT & .752 & .57 & 1413.963 & $<.001$ \\
\hline & AYU.APR & .556 & .31 & 485.234 & $<.001$ \\
\hline & VAL.ESF & .461 & .21 & 293.042 & $<.001$ \\
\hline \multirow{3}{*}{ PROGR } & DES.INT & .584 & .34 & 562.162 & $<.001$ \\
\hline & AYU.APR & .535 & .28 & 412.208 & $<.001$ \\
\hline & VAL.ESF & .438 & .19 & 257.227 & $<.001$ \\
\hline \multirow{3}{*}{ ESFUE } & DES.INT & .426 & .18 & 240.814 & $<.001$ \\
\hline & AYU.APR & .418 & .18 & 229.930 & $<.001$ \\
\hline & & .344 & .12 & 145.437 & $<.001$ \\
\hline
\end{tabular}

Como se observa en la Tabla 6, cada uno de los factores contextuales (el profesorado de Inglés Despierta Interés, Ayuda en el Aprendizaje y Valora el Esfuerzo) emergieron como variables predictivas de los factores disposicionales (Interés hacia la Asignatura, Progresión en el Aprendizaje y Esfuerzo en el Aprendizaje).

\section{DISCUSIÓN}

La estructura factorial de las escalas IEPA y AYES asociadas a la asignatura de Inglés como Lengua Extranjera de Enseñanza Secundaria, Bachillerato y Formación Profesional ha sido el objetivo central del presente estudio. El cálculo se ha hecho mediante un análisis factorial exploratorio o semiconfirmatorio, que con suficiente apoyo empírico ha ofrecido una distribución en tres factores para cada una de las escalas. El resultado de validez y fiabilidad ratifica el obtenido por Cecchini et al. (2014) en las asignaturas de Matemáticas, Lengua y Literatura y Educación Física validadas con estudiantes de Educación Secundaria Obligatoria.
Otro de los objetivos ha sido comprobar si las variables personales, edad y sexo, pueden inferir en los factores resultantes. En el presente estudio se ha comprobado que las mujeres puntúan significativamente más alto en interés y esfuerzo en la asignatura analizada, hecho que coincide con resultados obtenidos por Rodríguez-Pérez (2014), quien indica que las mujeres presentan mayor predisposición y una actitud más positiva hacia el aprendizaje.

En lo referente a determinar si las variables contextuales pueden ser predictivas de las variables disposicionales, se ha podido comprobar que el contexto donde se desarrolla el proceso de enseñanza del idioma, entendido como la percepción del alumnado sobre las estrategias de los docentes, en cuanto a la ayuda prestada en el proceso, las valoraciones positivas y la motivación, predice que los estudiantes se interesen, se esfuercen y se sientan más competentes en la asignatura de inglés. Los resultados apuntan en la misma dirección otros autores, a saber, Rubio y Martínez (2008), en un trabajo sobre la influencia de los aspectos educativos en el 
aprendizaje, indican que la práctica docente es un factor decisivo en el desarrollo de las habilidades para el aprendizaje de los idiomas; Bartau, Azpillaga y Joaristi (2017), también confirman que la metodología de enseñanza y los recursos educativos utilizados durante el proceso de enseñanzaaprendizaje constituyen un factor clave para alcanzar la eficacia. Salvado (2020) explica la importancia de poner a los estudiantes en situación de experimentar emociones positivas, ya que este hecho favorece la atención y la motivación hacia el aprendizaje. Muñoz, Lira, Lizama, Valenzuela y Sarlé (2019), centran la atención en la importancia y necesidad de que los docentes en formación experimenten experiencias satisfactorias que potencien su capacidad creativa para la implementación de actividades lúdicas como el propósito motivador. Barragan, Colcha y Herrera (2019), concluyen que si los docentes y estudiantes mantienen una actitud positiva y de disfrute durante el proceso de enseñanzaaprendizaje, favorece el aprendizaje de un idioma.

A la vista de los resultados obtenidos en este estudio, cabe destacar que la percepción de los contextos donde se desarrolla la enseñanza no muestra diferencias estadísticamente significativas entre sexos. Este resultado refuerza que la formación pedagógica del profesorado es un elemento decisivo para la enseñanza del idioma en estas edades. Dicho esto, y de acuerdo con las opiniones de varios autores (González-Pienda, González, Nuñez, \&Valles, 2002; Lozano, 2005; Martínez-Geijo, 2007), los docentes deben ser conocedores de técnicas de enseñanza individualizadas, donde se tenga en cuenta al estudiante como sujeto activo. Si además se potencia el sentido del humor en las aulas, se producirán avances significativos en el proceso de enseñanzaaprendizaje (Carbonero, Román, MartínAntón, \& Reoyo, 2009).

Durante la realización del trabajo se han detectado carencias que han acotado los resultados del mismo. Una importante limitación ha sido no considerar otras variables para reforzar los resultados, como por ejemplo el rendimiento académico de los estudiantes encuestados, aspectos relativos a su dificultad para interactuar en las clases o su autoestima y autoconcepto. No obstante, este estudio evidencia una continuidad en la investigación y lo dicho debe tenerse en cuenta para perfeccionar el trabajo y reforzar los resultados. Un posible futuro estudio podría ser realizar el Análisis Factorial Confirmatorio para ratificar los resultados, y una réplica del aquí presentado en una muestra similar específica a nivel de Formación Profesional, ya que en el presente trabajo la representación de este tramo educativo ha sido escasa.

También se deberían corroborar la validez y fiabilidad de las escalas en el nivel universitario, lo que supondría ampliar la categoría de las edades a la adolescencia tardía, entre los 18 y los 25 años, pues hasta el momento solamente se han aplicado en preadolescentes y adolescentes. Sería importante también legitimar las escalas en otros países de habla hispana, y traducirlas y adaptarlas para su validación en otros idiomas. Finalmente, a la vista de los resultados obtenidos sobre la influencia que ejerce el contexto donde se desarrolla el aprendizaje en el interés, esfuerzo y competencia percibida por los estudiantes, se abre la necesidad de realizar un estudio experimental, con grupo control y grupos experimentales, ofreciendo diversos contextos, con el objetivo de comprobar cuáles son los métodos o estrategias que mejor faciliten y motiven el aprendizaje de otras lenguas, y en qué entorno los estudiantes se sientan más seguros.

\section{- Conflicto de intereses.}

Los autores declaran no tener ningún conflicto de intereses.

\section{REFERENCIAS}

Balaguer, I., Castillo, I., \& Duda, J. L. (2001). Perspectivas de meta de los adolescentes en el contexto académico. Psicothema, 13(1), 79-86.

Bandalos, D. L., \& Finney, S. J. (2010). Factor Analysis: Exploratory and Confirmatory. En G. R. Hancock \& R. O. Mueller (Coords.). Reviewer's guide to quantitative methods. New York: Routledge. 
Barragán, R., Colcha, E. I., \& Herrera, Z. V. (2019). La motivación y el uso de estrategias en la enseñanza - aprendizaje para docentes del idioma inglés. Ciencia Digital, 3(3.2.1), 310-322. https:// doi.org/10.33262/cienciadigital. v3i3.2.1.857

Bartau, I., Azpillaga, V., \& Joaristi, L. M. (2017). Metodología de enseñanza en centros eficaces de la Comunidad Autónoma del País Vasco. Revista de Investigación Educativa, 35(1), 93-112. https://doi.org/10.6018/rie.35.1.225141

Bastidas, J., \& Muñoz, G. (2020). Factores que influyen en el aprendizaje del inglés de los bachilleres de Pasto, Colombia. Folios, 51, 163-181.doi: 10.17227/folios.51-8676

Carbonero, M. A., Román, J. M., MartínAntón, L. J., \& Reoyo, N., (2009). Efecto del programa de habilidades docentes motivadoras en el profesorado de secundaria. Revista de Psicodidáctica, $14(2), 229-244$.

Burgueño, R., Sicilia, Á., Alcaraz-lbáñez, M., Lirola, M. J., \& Medina-Casaubón, J. (2020). Efectos del contenido de meta docente y la regulación motivacional académica sobre la creencia de eficacia docente del profesorado en formación inicial. Educación XX1, 23(1), 103-124. https://doi:10.5944/educXX1.23672.

Cecchini, J. A., González-Pienda, J. A. Méndez-Giménez, A., Fernández-Río, F. J. Fernández-Losa, J. L., \& González, C. (2014). Assessment of dispositional and contextual variables in educational settings: IEPA and AYES scales, Psicothema, 26(1), 76-83. https://doi:10.7334/ psicothema2013.113.

Crookes, G., \& Scmidt, R. W. (1991). Motivation: Reopening the Research agend. Language learning, 41, 469-512. https://doi:10.1111/i.1467-1770. 1991. tb00690.x

Curran, C. A. (1976). Counseling-learning in second languages. Apple River, IL: Apple River Press.

Dörney, Z. (2001). Motivational strategies in the language classroom. Cambridge: Cambridge University Press.

Duda, J. L., \& Nicholls, J. G. (1992). Dimensions of achievement motivation in schoolwork and sport. Journal of Educational Psychology, 84, 290-299.

Elosua, P., \& Zumbo, B. (2008). Coeficientes de fiabilidad para escalas de respuesta categórica ordenada. Psicothema, 20(4), 896-901.

Ferrando, P. J. \& Anguiano-Carrasco, C. (2010). El análisis factorial como técnica de investigación en psicología. Papeles del Psicológo, 31 (1), 18-33.

Ferrando, P. J., \& Lorenzo-Seva, U. (2014). El análisis factorial exploratorio de los ítems: algunas consideraciones adicionales. Anales psicología, 30(3), 1170-1175. https:// doi:10.6018/analesps.30.3.199991.

Finn, J., \& Rock, D. (1997). Academic success among students at risk for school failure. Journal of Applied Psychology, 82(2), 221-234. https://doi.org/10.1037/00219010.82 .2 .221

Forero, C. G, Maydeu-Olivares, A., \& Gallardo-Pujol, D. (2009). Factor analysis with ordinal indicators: A Monte Carlo study comparing DWLS and ULS estimation. Structural Equation Modeling, 16, 625-641. Gabarron, A. (2019). La enseñanza de lenguas extranjeras y la Teoría de las Inteligencias Múltiples de Gardner. Reflexiones sobre la importancia de la emoción en el aprendizaje de lenguas extranjeras. Magister, 37 (2), 1924.

Gardner, R. (1985). Social psychology and second language learning: The role of attitudes and motivation. Londres: Edward Arnold.

Gardner, R., Tremblay, P. F., \& Masgoret, A. (1997). Towards a full model of second language learning; an empirical investigation. Modern Language Journal, 81, 344-362.

Gómez-Ortiz, O., Romera, E. M., \& OrtegaRuiz, R. (2017). La competencia para gestionar las emociones y la vida social y su relación con el fenómeno del acoso y la violencia escolar. Revista Interuniversitaria de formación del Profesorado, 88(31.1), 27-38.

González-Pienda, J. A., González, R., Nuñez, J. C., \& Valle, A. (2002). Manual de psicología de la educación. Madrid: Pirámide.

Guillen, F., Pérez-Luzardo, J., \& Arnaiz, P. (2013). Relación entre motivación y 
optimismo disposicional en aprendices de inglés como lengua extranjera en un contexto universitario. Revista de Educación, No Extraordinario, 104-128. https:// doi:10.4438/1988-592X-RE-2013-EXT-243 Kline, P. (1999). The handbook of Psychological Testing. London: Routledge

Krashen, S. D. (1982). Principles and practice in second language acquisition. Oxford: Pergamon Press.

Ley orgánica para la mejora de la calidad educativa (LOMCE) (Ley Orgánica 8/2013, 9 de diciembre). Boletín Oficial del Estado, $n$ - 295, 2013, 10 diciembre.

Lightbown, P. M., \& Spada, N. (2013). How languages are learned (4th edition). Oxford: University Press.

Lozano, A. (2005). Estilos de aprendizaje y enseñanza. México: Trillas.

Madrid, D. (1999). La investigación de los factores motivacionales en el aula de idiomas. Granada: Universidad de Granada.

Martínez-Geijo, P. (2007). Aprender y enseñar: Los estilos de aprendizaje y de enseñanza desde la práctica de aula. I.C.E. Universidad de Deusto: Mensajero, SAU.

Mas, C., \& Medinas, M. (2007). Motivaciones para el estudio en universitarios. Anales de Psicología, 23(1), 17-24.

Muñoz, C., Lira, B., Lizama, A., Valenzuela, J., \& Sarlé, P. (2019). Motivación docente por el uso del juego como dispositivo para el aprendizaje. Interdisciplinaria, 36(2), 233249.

Muthén, B., \& Kaplan D. (1992). A comparison of some methodologies for the factor analysis of non-normal Likert variables: A note on the size of the mode. British Journal of Mathematical and Statistical Psychology, 45, 19-30. https:// doi.org/10.1111/i.2044-8317.1992. tb00975.x

Nevid, J. (2013). Psychology: concepts and applications. Belmont, CA: Wadsworth Cengage Learning.

Real Academia Española (2001). Diccionario de la lengua española (22.a ed.). Consultado en http://www.rae.es/rae.html
Riemer, C., \& Schlak, T. (2004). Der Faktor motivation in der fremdsprachenforschung, Zeitschrift für Interkulturellen Fremdsprachenunterricht, 9(2), 1-3.

Rodríguez-Pérez, N. (2014). Creencias y representaciones de los profesores de lenguas extranjeras sobre la influencia de los factores motivacionales y emocionales en los alumnos y en las alumnas. Porta Linguarum, 21, 183-197.

Rost-Roth, M. (2001). Zweitsprachenenwerb als individueller rozess IV: affektive variablen. Berlín: Walter de Gruyter.

Rubio F. D., \& Martínez M. (2008). La comunicación oral del inglés en España: influencia de los aspectos educativos y mediáticos en el éxito del aprendizaje. Revista de Enseñanza Universitaria, 32, 51-63.

Salvado, J. J. (2020). Potencialidad del uso del lenguaje positivo en el proceso de enseñanza-aprendizaje. Tecnología, ciencia y educación, 15, 81-103.

Sánchez, L. (2017). Las emociones al servicio del aprendizaje de una segunda lengua: propuesta para el desarrollo de la competencia oral a través de la educación emocional (Trabajo Fin de Grado). Valladolid: Universidad de Valladolid.

Schmider, E., Ziegler, M., Danay, E., Beyer, L., \& Bühner, M. (2010). Is it really robust? Reinvestigating the robustness of ANOVA against violations of the normal distribution assumption. Methodology, 6(4), 147-151. https://doi: 10.1027/1614-2241/a000016

Timmerman, M. E., \& Lorenzo-Seva, U. (2011). Dimensionality assessment of ordered polytomous items with parallel analysis. Psychological Methods, 16(2), 209-220. https://doi: 10.1037/a0023353

Tragant, E., \& Muñoz, C. (2000). La motivación y su relación con la edad en un contexto escolar de aprendizaje de una lengua extranjera. En C. Muñoz (Coord.) Segundas lenguas. Adquisición en el aula (pp 81 -105). Barcelona: Ariel Lingüística. 